\title{
The ART of Cosmological Simulations
}

\author{
Stefan Gottlöber and Anatoly Klypin
}

\begin{abstract}
We describe the basic ideas of MPI parallelization of the N-body Adaptive Refinement Tree (ART) code. The code uses self-adaptive domain decomposition where boundaries of the domains (parallelepipeds) constantly move - with many degrees of freedom - in the search of the minimum of CPU time. The actual CPU time spent by each MPI task on a previous time-step is used to adjust boundaries for the next time-step. For a typical decomposition of $5^{3}$ domains, the number of possible changes in boundaries is $3^{84} \approx 10^{40}$. We describe two algorithms of finding minimum of CPU time for configurations with a large number of domains. Each MPI task in our code solves the N-body problem where the large-scale distribution of matter outside of the boundaries of a domain is represented by relatively few temporary large particles created by other domains. At the beginning of a zerolevel time-step, domains create and exchange large particles. Then each domain advances all its particles for many small time-steps. At the end of the large step, the domains decide where to place new boundaries and re-distribute particles. The scheme requires little communications between processors and is very efficient for large cosmological simulations.
\end{abstract}

\section{Introduction}

During the last 10 years new extensive observations of the Universe were made using both ground-based telescopes and space instruments. These measurements have provided new insights into the structure of the Universe on various scales. A wide

Stefan Gottlöber

Astrophysical Institute Potsdam, An der Sternwarte 16, 14482 Potsdam, Germany e-mail: sgottloeberdaip.de

Anatoly Klypin

Astronomy Department, New Mexico State University, MSC 4500, P.O.Box 30001, Las Cruces, NM, 880003-8001, USA e-mail:aklypin@nmsu.edu 
range of the electromagnetic spectrum emitted by cosmic objects has been studied. The wavelengths extend from very long radio wavelengths to energetic gamma rays. This observational progress has been accompanied by considerable effort in our theoretical understanding of the formation of different components of the observed structure of the Universe: galaxies and their satellites, clusters of galaxies, and super-clusters. A substantial part of this theoretical progress is due to the improvment of numerical methods and models, which mimic structure formation on different scales using a new generation of massive parallel supercomputers.

The collective effort of observers and theorists brought into being the standard cosmological model, which is based on the idea that some kind of dark energy contributes about $70 \%$ of the total energy-density of the spatially flat Universe. The simplest form of the dark energy is the cosmological constant, which was introduced in 1917 by Albert Einstein in his paper about the cosmological solutions of the field equations of general relativity. The remaining $30 \%$ of energy density consists of matter. About $85 \%$ of this matter is made of unknown dark matter particles, which interact only gravitationally. Only the remaining $15 \%$ is the contribution of "normal" (baryonic) particles, well known to particle physicists. This means that at present we know the nature of only $5 \%$ of the total energy in the universe, the remaining $95 \%$ is not yet understood.

The main process responsible for the formation of observed structures is the gravitational instability. The initial seeds, which eventually became galaxies and superclusters and all the other structures, came from the quantum fluctuations generated during the early inflationary phase. The power spectrum of these primordial fluctuations has been confirmed by measurements of the temperature fluctuations of the cosmic microwave background radiation. These temperature fluctuations tell us the magnitude of the small density fluctuations in the Universe when it was about 300000 years old. One of the key features of the standard model is its simplicity. The expansion rate and the clustering properties are described by only few parameters which are measured at present already with quite high accuracy.

Since about $85 \%$ of the matter consists of only gravitationally interacting particles this dark matter forms the backbone structure for all objects in the Universe from clusters of galaxies to dwarf satellite galaxies. Baryonic matter falls into the potential wells created by the dark matter and forms luminous objects. The nonlinear evolution of cosmological fluctuations can be studied only numerically. The details of galaxy formation must be followed using hydrodynamic simulations. However, many features can already be studied by semi-analytical methods which are based on the evolution of dark matter halos as measured in the dark matter simulations. Thus, numerical simulations are an important tool to understand the formation of structure in the Universe.

The requirements for modern cosmological simulations are extreme: a very large dynamical range for force resolution and many millions of particles are needed. These requirements are just a reflection of the vast range of masses and spatial scales in real astronomical objects. For example, from dwarf galaxies to galaxy clusters the mass spans about 7 orders of magnitude. The range of scales is also enormous: from 
the inner structure of galaxies (sub-kiloparsec scales) to cosmological distances of hundreds of megaparsecs.

\section{The Adaptive Refinement Tree (ART) code}

\subsection{History}

To follow the evolution of the dark matter in the expanding Universe one has to solve the coupled system of the Poisson and Vlasov equations. These are the standard equations of motion for particles interacting via gravity. Since the number of particles representing the distribution of dark matter is very large, direct integration of these equations is not possible. Over the last three decades several methods have been developed to solve simultaneously the Poisson equation for the gravitational potential and Newton's equation for the acceleration for large numbers of particles (see [7] for an overview).

The Adaptive Refinement Tree (ART) code was build by a number of people starting 1979. In its first version it was a particle-mesh code written by A. Klypin in collaboration with A. Doroshkevich and S. Shandarin (then at the Institute of Applied Mathematics in Moscow). At that time the code used a cubic mesh to assign density and to solve the Poisson equation. The Cloud-In-Cell algorithm was used to find the density. Due to the limited computer resources in the early eighties the first version of the code could handle only $32^{3}$ particles.

In 1995 A. Khokhlov [8] developed the Fully Threaded Tree algorithm for Adaptive Mesh Refinement. He provided routines to handle data structures in the new Adaptive Mesh Refinement (AMR) scheme. Using the previous codes and Khokhlov's new algorithm, in 1996/97 A. Kravtsov [10, 11] wrote the first version of the ART code. This version of the code used the OpenMP parallelization.

Because the parallelization of ART with OpenMP is not very efficient, there was a need to substantially increase the scalability of the code in order to use it on massively parallel computers. Starting 2000, we developed MPI versions of the code based on the OpenMP code. The first hybrid MPI+OpenMP code was written to simulate the evolution of 13 galaxy clusters using 8 nodes of the Hitachi supercomputer at LRZ. The code was run as a farm of non-communicating OpenMP jobs. On each of the nodes the OpenMP parallelized code was running on 8 CPUs.

At that time the code also was modified to treat particles of different masses and to have high resolution in some specific regions of the computational box. For example, in one of the simulations we selected high density regions within a $80 h^{-1} \mathrm{Mpc}$ box and covered the regions with many small mass particles, whereas the large scale tidal field was represented by massive particles in the rest of the computational box. To avoid numerical problems between the high- and low- resolution regions, several layers with particles of increasing mass were added. Different high mass resolution areas were given different MPI tasks. The load balance in this case is not very good 
because the evolution proceeds differently in different simulations. However, this is not a big problem because different regions of the simulations could be in different stages of evolution and we can use fewer MPI tasks once some of the jobs are finished.

In 2002-2003 we developed a full MPI+OpenMP code. The motivation to have a hybrid MPI+OpenMP code is to address two issues: (a) The OpenMP parallelization is not very efficient for large number of processors and the code scaling depends on particular computer architecture. As of 2007, the code scales well up to 4 processors on shared memory computers such as Altix or SP5. For example, on a quad Opteron systems the speedup is 1.8 for two processors and 2.4 for four processors. The main bottleneck for OpenMP is the data locality. Thus, MPI is necessary, if we want to use more processors. (b) Significant memory is required by the code, and OpenMP provides the way to access a larger memory: when we use OpenMP, all the memory of a node is accessible for the code. Therefore, we typically use 2-4 processors per MPI task depending on the memory requirements of our computational problem and on the computer architecture.

Since 2004 the MPI version of the code was used on different computers like the Hitachi and Altix of the Leibnizrechenzentrum Munich, the Altix of the NASA Ames, the Opteron cluster at AIP, the p690 cluster at Julich and the MareNostrum computer in Barcelona. Depending on the requirements of our tasks and the computer architecture, we used 32 to 510 processors for our simulations.

Moore's law is roughly reflected also in the evolution of the ART code: It's very first version could handle $32^{3}$ particles, whereas 20 years later the MPI version handles $1024^{3}$ particles, a factor of $2^{15}$ increase. Moore's law predicts a factor of $2^{13}$.

At present ART is a family of codes, which sprouted up from the same AMR code written by A. Kravtsov [10, 11]:

- OpenMP-only N-body code. This has been often used for simulations of isolated stellar dynamical systems [20, 2], for a computational box with up to $256^{3}$ particles [3], or for a single high-resolution region in a large computational box [6].

- MPI+OpenMP N-body code. This code is used for large cosmological simulations.

- OpenMP N-body+hydro code. Examples of using the code include simulations of clusters of galaxies [13, 16], large-scale distribution of gas in the Local Supercluster [12], and formation of galaxies [1].

- MPI N-body+hydro code. This was written by D. Rudd and A.Kravtsov [18]. N. Gnedin incorporated radiative transfer code into the ART hydro code [5].

Here we discuss parallelization of the MPI+OpenMP N-body code. 


\subsection{Method}

The code starts with a regular cubic grid, which covers the entire computational volume and defines the minimum resolution of the simulation. If the mass in a cell exceeds some threshold, the cell can be split into eight cells each half the size. If mass in any of the new cells is still above the threshold, the cell can be split again. In order to avoid too large jumps in the sizes of adjacent cells, the code enforces splitting of cells in such a way that refinement levels of any adjacent cells differ by not more than one level. In other words, if $n$ is the level of a cell, than its immediate neighbor may be only a cell on levels $n-1, n, n+1$. The code constructs meshes of arbitrary shape covering equally well both elongated structures (such as filaments and walls) and roughly spherical dark matter halos. The meshes are modified to adjust to the evolving particle distribution by creating new cells and by destroying old ones. The threshold for the refinement is a free parameter, which is typically 2-4 particles in a cell. The algorithm of the refinement is very flexible and can be easily adjusted for a particular problem. For example, we can allow the construction of the refinements only in some specified area of the computational volume. This is done by constructing a map of refinements: only cells marked for refinement are allowed to be split.

The ART code integrates trajectories of collisionless particles by employing standard particle-mesh techniques to compute particle accelerations and advance their coordinates and velocities in time using the leap-frog scheme. The time-step in ART code depends on the resolution level: the higher the level (and the density), the smaller the time-step. The time-step decreases by a factor of two with each refinement level. In cosmological simulations the refinement can reach 10 levels, which gives 1024 times smaller time-steps as compared with the zero-level time-step. Typically a cosmological simulation has between 300 and 1000 zero-level time-steps or even more in the case of very high resolution runs. The ART code should run with sufficiently small time-step so that the maximum displacement does not exceed a fraction of a cell. Typically, it should be below $0.20-0.25$. This corresponds to the rms displacement in the range $0.05-0.1$. If the maximum displacement goes above unity, the code may become unstable and it should be restarted from the very beginning with a smaller time-step.

To solve the Poisson equation the code uses the FFT solver at the zero-level of refinement and a multilevel relaxation method with odd-even Successive Overrelaxation with Chebyshev acceleration at each non-zero level.

\subsection{The MPI version of the code}

The basic idea of MPI parallelization of the ART code is to decompose the simulation volume into rectangular domains. Each MPI task handles one domain and employs the OpenMP version of the code. Communications between MPI tasks occur only at the beginning of each zero-level time-step. Each MPI task receives in- 

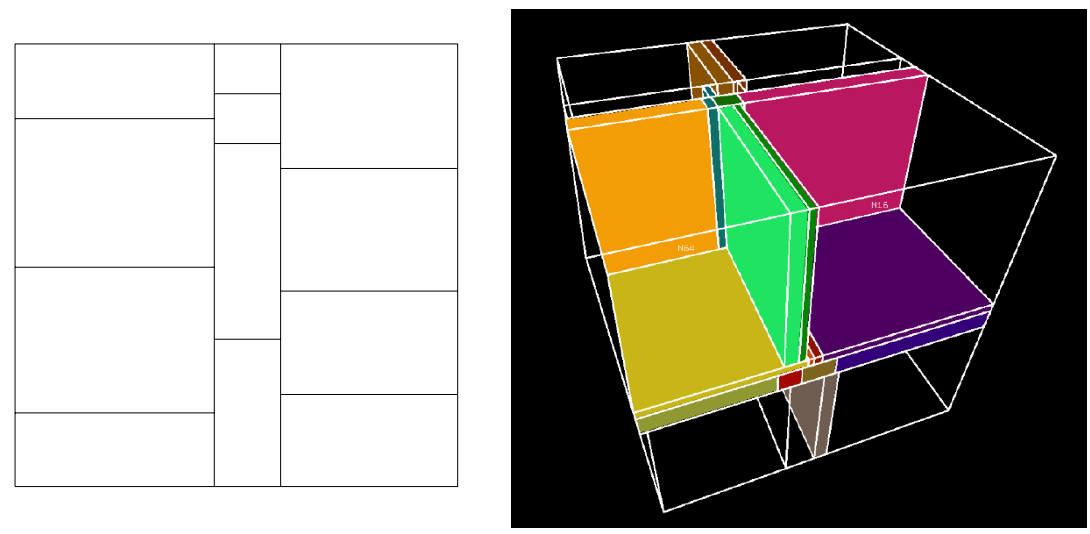

Fig. 1 Left: Example of the $3 \times 4$ domain decomposition in 2D. Boundaries of domains are aligned in $\mathrm{x}$-direction, but there is no alignment of domains in y-direction. The total number of degrees of freedom is 11 . Each boundary can be adjusted in order to minimize the maximum CPU of MPI tasks. Right: Domain decomposition in 3D for a high resolution simulation, which was dominated by a single halo in the center of the box. The code was configured to have $4 \times 4 \times 4$ domains. This is an extreme case with some domains being very elongated. Typical simulations have more even distribution of domains. There is no problem in solving the force of gravity even in these extreme conditions: this is what AMR-type codes are designed for. Yet, the code is not efficient because most of the CPU time goes to the central region and there is little left for the rest of the domains.

formation about the mass distribution and velocity field in the whole computational volume. This information is accurate enough to advance particles handled by the task to the next time-step. The information comes in the form of massive particles, which represent mass distribution and velocity away from the domain of the MPI task. At the end of the zero-level step these additional particles are discarded and the whole process starts again.

\section{Domain decomposition}

We use rectangular domains for MPI parallelization. The whole simulation volume a cube - is split into non-overlapping fully covering parallelepipeds. The boundaries of the parallelepipeds can move as time goes on in order to equalize the load of different MPI tasks. In Fig. 1 left panel we show an example of a possible splitting of the computational volume in the two-dimensional case. Note, that boundaries in $\mathrm{x}$-direction are aligned, but they are not aligned in $\mathrm{y}$ - (and in 3D in z-) directions.

Each boundary can move only at the beginning of a zero-level time-step. Once the zero-level time-step is completed, information on CPU time consumed by different 
MPI tasks is used to adjust the boundaries to improve the load balance. At present boundaries of the domains can have only discrete positions: they can only be placed at boundaries of the zero-level mesh. The number of degrees of freedom to move domains can be very large. It depends on the number and the configuration of the domains. For the example in Fig. 1 left panel there are 3 domains in $x-$ and 4 domains in $\mathrm{y}$ - direction. In the general case of the division of the volume by $n_{x}$ domains in the x-direction and $n_{y}, n_{z}$ in y-and z-directions, the number of degrees of freedom is $\left(n_{x}-1\right)\left[\left(n_{y}-1\right)\left(n_{z}-1\right)+\left(n_{y}-1\right)+1\right]$.

There are different ways of using this large number of degrees of freedom to improve the load balance. The current version of ART provides two routines for load balance. The first routine assumes that within each domain $(i, j, k)$ the CPU time $T(i, j, k)$ is homogeneously distributed. In other words, the density of the CPU time is treated as a piece-wise constant function. Each boundary can have only three positions: current, current plus one zero-level cell, and current minus one zero-level cell. The code loops through a very large set of configurations of boundaries (up to 60000 ) and finds the minimum value of the maximum expected CPU time in the domains. The minimization routine is very fast - it takes only a fraction of a second to find the minimum. By design, it minimizes the maximum CPU time of a MPI task. This works reasonably well when the system is evolving slowly and the maximum is not jumping from one area to another. The constraint that boundaries can move only by one cell works well for systems, which evolve slowly and for which the load balance is already reasonable. In this case the code tunes the load balance.

For early stages of evolution and for quickly evolving systems ART uses a second algorithm of load balancing. The algorithm is to equalize the load balance along each direction. It starts with the x-direction. All CPU times are summed up for all tasks, which have the same $\mathrm{x}$-boundaries. In Fig. 1, right panel this gives three numbers each being a sum of CPU time of domains in the y-direction with the same $\mathrm{x}$-boundaries. We can describe each domain by a triplet of integers $(i, j, k)$, where integers are in the ranges $\left[1, n_{x}\right],\left[1, n_{y}\right],\left[1, n_{z}\right]$. The procedure of summing up CPU times gives $\sum_{j, k} T(i, j, k)$. Assuming that the CPU time is constant inside boundaries of each domain, we get a piece-wise linear function of CPU time from $x=0$ to given $x$. We can place new $x$-boundaries of domains in such a way that each sum of domains with given $x$-boundaries has the same CPU time: $\sum_{j, k}(i, j, k)=T(i)=$ const. We then repeat this procedure for $y$ - and $z$-directions.

The configuration of domains - how many in each direction and the boundaries of the domains - is in a configuration file. The Fig. 11 right panel gives an example how the code can adjust boundaries of domains in its effort to load-balance the run. In this extreme example there was one large halo close to the center of the computational box and few smaller halos and filaments around it. The code was using $4 \times 4 \times 4$ domains. After some period of evolution, the code evolved in such a way that it had eight large domains in the corners of the simulation box, which contained only a small number of particles. Most of the computational effort was in the smaller domains, which cover the central region of the box where the massive halo and few smaller ones have formed. 


\section{Exchange of information between MPI tasks}

At the beginning of each zero-level time-step the MPI tasks exchange information. This is very infrequent. The main idea for the information exchange is the same as in TREE codes: the mass distribution at large distances can be approximated roughly when forces are calculated. In the ART code this idea is implemented by creating increasingly more massive particles with increasing distance from the boundaries of a domain handled by an MPI task. In addition, every domain is surrounded by a buffer zone, from which it receives primary (small) particles. Particles are not averaged in this buffer zone. The width of the buffer is a parameter. We typically use (0.5-1) of the zero-level cell.

Thus, each MPI task has three types of particles: (1) primary particles of low mass, (2) low mass particles in the buffer zone, and (3) progressively more massive temporary particles. The set of all particles in each domain covers the whole computational volume. Each MPI task handles the whole volume and there is no other exchange of information between MPI tasks until the end of the given zero-level time-step. Only at the beginning of a zero-level time-step the temporary particles are created and exchanged. During one zero-level time-step each MPI task advances all its particles (primary as well as temporary).

Once the time-step is finished, the CPU time consumed by every MPI task is gathered by the root task which decides how to move the boundaries of the domains in order to improve the load balance. The primary particles are re-distributed so that they reside on tasks, which handle the domains. Then the process starts again: exchange of buffer particles, creation and sending of temporary particles.

Massive particles are created in the following way. Each domain (a parallelepiped) is covered by a hierarchy of grids. The first grid has cell size equal to the zero-level mesh. The second mesh has cells twice the size of the zero level, the third mesh has cells twice the size of the second level, and so on for higher level meshes. There are 4 levels of meshes for construction of large temporary particles. We find mass, average velocity, and center of mass of all primary particles in each cell for each mesh. This creates temporary massive particles, which are sent from one domain to another to trace the external gravity field. The level of grid, from which a temporary particle is taken, depends on the distance to the boundary of the domain to which the particle will be sent: the larger the distance the higher is the level of the grid. If the zero-level mesh has $n_{g}$ cells along each direction and $L$ is the length of the computational box, then the zero-level cell has size $d_{0}=L / n_{g}$. This length provides a scale for the auxiliary meshes. Within a shell of 8 zero-level cells, which surrounds a given domain, the mesh used for creating temporary particles is $d_{0}$. The next shell of 8 cells gives larger particles taken from the second level mesh. The averaging size of the mesh is $2 d_{0}$. For the third shell of 8 cells the averaging size is $4 d_{0}$. Finally everything else is covered with $8 d_{0}$ cells. (The code is written for arbitrary number of mesh levels.)

We can estimate the number of temporary particles for each domain by assuming that the distribution of mass is not too inhomogeneous. In this case each domain has approximately $K_{i}=n_{g} / n_{i}$ zero-levels cells in each $i-t h$ direction. Further assuming 
that the number of domains in each direction is the same $n_{x}=n_{y}=n_{z}=n$, we estimate the number of temporary particles $N_{\text {temp }}$ on all levels:

$$
N_{\text {temp }}=\frac{n_{g}^{3}}{8^{3}}+7 \sum_{j=1,3}\left(\frac{K+16 j}{2^{j}}\right)^{3}-K^{3},
$$

where $K=n_{g} / n$ is the number of zero-level cells in 1D in each domain. For typical values $n_{g}=256, n=4-6$, we get $N_{\text {temp }}=(2-4) \times 10^{5}$. Most of the particles are coming from the first (high resolution) shell.

In the same fashion we also can estimate the number of primary particles in the buffer zone:

$$
N_{\text {buffer }}=\frac{N_{\text {part }}}{N_{\text {domains }}}\left[\left(1+\frac{2 d x \cdot n}{n_{g}}\right)^{3}-1\right],
$$

where $N_{\text {part }}$ is the total number of the primary particles in all domains, $N_{\text {domains }}=n^{3}$ is the number of the MPI tasks, and $d x$ is the width of the buffer zone in units of the zero-level cell. For typical numbers $N_{\text {part }}=1024^{3}, N_{\text {domains }}=6^{3}, d x=0.5$, and $n_{g}=$ 256, we get $N_{\text {buffer }}=3.5 \times 10^{5}$. This should be compared with number of primary particles of the MPI task $N_{\text {main }}=\frac{N_{\text {part }}}{N_{\text {domains }}} \approx 5 \times 10^{6}$. This means that the overhead of the domain decomposition method is about 10 percent. The actual overhead can be larger. For large cosmological simulations with sizes of simulation boxes $100 \mathrm{Mpc}$ and larger we actually measured overheads close to the theoretical 10 percent, when the number of domains was $\sim 5^{3}-6^{3}$ and the number of processors was 250-500. Simulations of small high-resolution regions embedded into a large computational boxes get less efficient as the size of the high resolution region gets closer to the size of a zero-level cell. Simulations of an isolated halo, which is only a few zero-level cells across do not scale and cannot be done efficiently with the percent version of the code. For the code to be efficient the rule of thumb is that the number of large virialized systems consuming most of the CPU should be larger then the number of MPI tasks. The problem with the scalability of very large single-object simulation is not specific to the ART code. To improve the scalability the decomposition should be done on high levels of the force refinement.

Using the estimates of the number of the particles, we can find how much data should exchanged between MPI tasks. Each particle in the simulation needs 6 numbers with double precision ( 8 bytes each) and three auxiliary single precision variables. The total is 60 bytes per particle. Thus the total amount of data each MPI task receives and sends is about $100 \mathrm{Mb}$ for the typical values presented above. Specifically, we use nine mpi_alltoallv calls to distribute the particles among the MPI tasks.

\section{Input - Output}

Each of the $N_{\text {MPI }}$ tasks reads its own files with information about parameters, coordinates, velocities, particle masses and ids and refinement levels of each particle. These files are stored in $N_{\text {MPI }}$ directories. When the code starts, every MPI task goes 

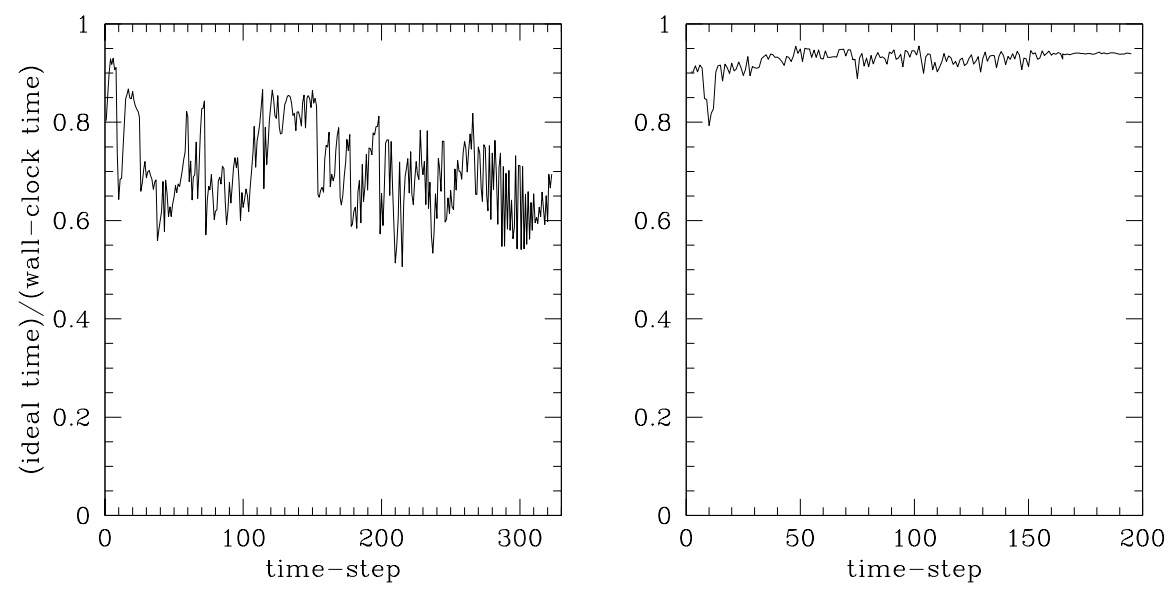

Fig. 2 Load balance for two runs with $1024^{3}$ particles. We show the ratio of the ideal CPU time to the wall-clock time used for each zero-level time-step. The ideal time is calculated as the sum of CPU time over all procesors divided over the number of processors. The wall clock time is the time of the slowest MPI task. Left: Computational box is $160 h^{-1} \mathrm{Mpc}$. In this case few large clusters dominate the evolution of the system and cause some load imbalance. The run was using 504 processors of the Altix system at LRZ Munich. Right: Load balance for a run with $1024^{3}$ particles in a $1000 \mathrm{~h}^{-1} \mathrm{Mpc}$ computational box. The code used 500 processors (125 MPI tasks with 4 processors in each task) of the Altix 4700 system at LRZ Munich and, starting with the time-step 150, on Columbia Altix 3700 system at Nasa Ames. Initial load imbalance (time-step about 20) occurs when the system starts to open refinement levels in different parts of the box. Once nonlinear structures appear all over the computational volumes, the code adapts and equalizes the load reasonably well.

to its subdirectory and reads its files. In the sense of structure, there is no difference between snapshot files and the re-start files so that one can easily restart from any earlier saved snapshot. In each subdirectory there are additional files that contain protocols of running the job, in particular the CPU time spent at each time-step by the given MPI task.

The root task writes an additional file, which provides details of the distribution of CPU time among different MPI tasks, it contains the maximum, minimum, and average CPU time per zero-level time-step in units of seconds as well as the CPU time used by each MPI task in units of maximum CPU time. This tells us about the load balance. As an example the load balance for a simulation of a computational box of $160 h^{-1} \mathrm{Mpc}$ side length with $1024^{3}$ particles is shown in Fig. 2, left panel. For this simulation we used 252 nodes with 2 CPU per node on the Altix of LRZ Munich. Fig. 2] right panel shows the load balance in another simulation with $1024^{3}$ particles. Due to the lower number of MPI tasks and the larger volume of the box (1000 $h^{-1} \mathrm{Mpc}$ side length) the averaged volume which each MPI task has to handle is much larger. Therefore, in this case the balance was significantly better.

Our scheme of MPI parallelization has one significant positive feature: it has very little communications. Communications happen only once every zero-level timestep, when the particles are re-arranged between different MPI tasks. For a typical 


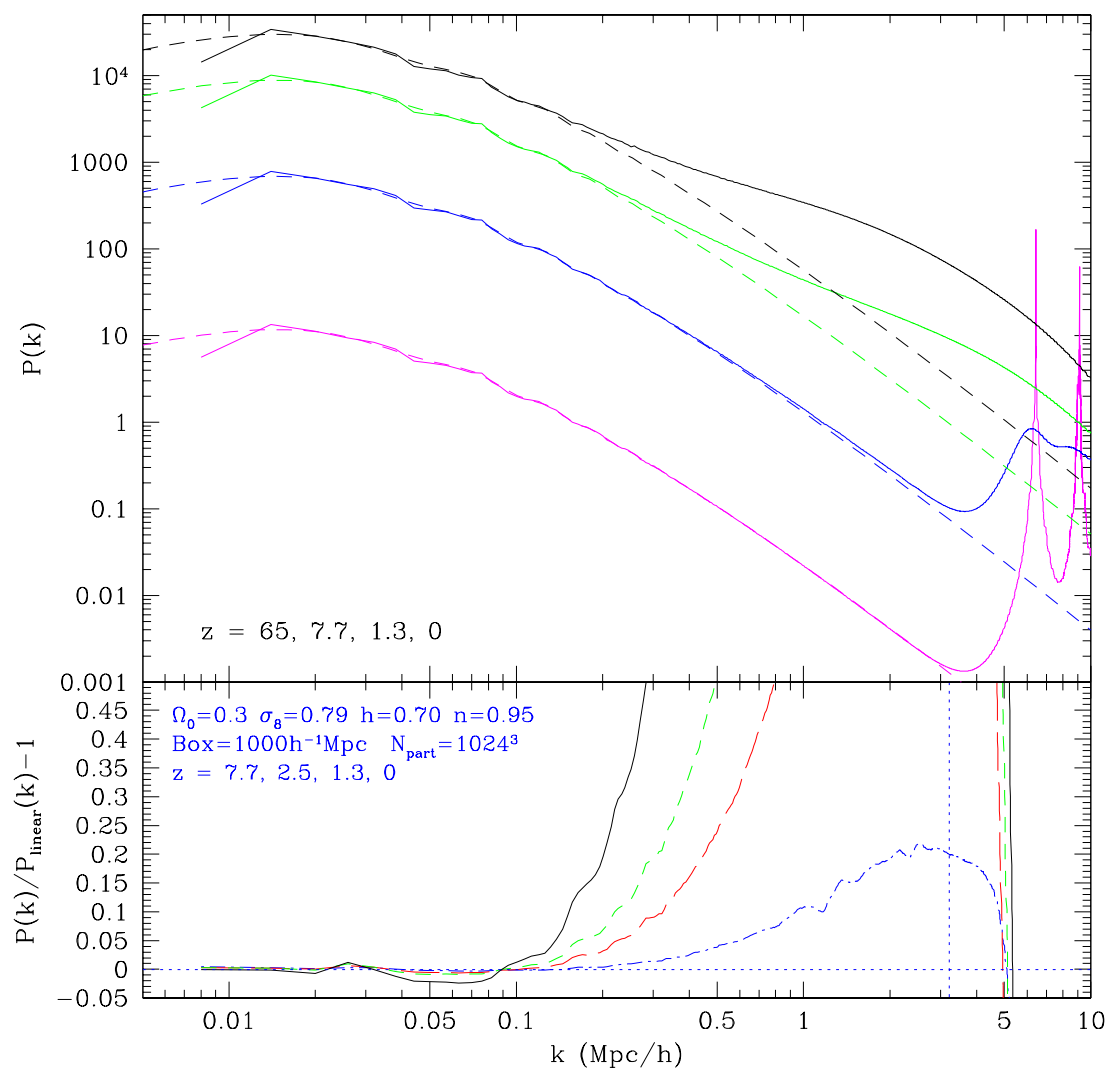

Fig. 3 Growth of perturbations in the $\Lambda \mathrm{CDM}$ model. The top panel shows the evolution of the power spectrum $P(k)$ in the simulation (full curves) as compared with the linear theory (dashed curves). From bottom to top the curves correspond to decreasing redshifts: the lowest curve is for the initial conditions $(z=65)$ and the top curve is for $z=0$. The deviation in the first harmonic (the smallest $k$ ) is due to small statistics of the longest waves. Two strong spikes at large $k$ 's are above the Nyquist frequency: the N-body code does not "see" them. Bottom panels show deviations from the predictions of the linear theory. Fluctuations on large scales (small $k$ 's) grow according to the linear theory. The dot-dashed $(z=7.7)$ and the long-dashed curves $(z=2.5)$ show that non-linear evolution increases the power spectrum on all scales proceeding from high $k$, where the non-linear effects are strongest, to low $k$, where the effects are weakest. The vertical dotted line shows the Nyquist frequency of particles. Perturbations with the frequencies above the Nyquist frequency do not grow in linear and in quasi-linear regimes.

simulation this happens 200-500 times during the whole run (so, every $30 \mathrm{~min}-1 \mathrm{hr}$ of wall-clock time for a large run). During that stage every MPI task receives about $100-500 \mathrm{Mb}$ of data. Then there will be no communications till the next time-step. The scheme has its overheads and limitations. CPU time required to handle particles in a narrow buffer zone around each domain is a loss. Massive particles, which 
represent external density field is also a loss, but the CPU required for them is very small: a fraction of a percent of the total CPU. As long as the number of particles in the buffer zone is small, the code works reasonably well. Thus, good load balance can be reached in large cosmological runs that cover the whole computational box with equal-mass particles. In case of multi-mass runs which resolve only a certain region of the box (as shown in Fig. 1, right panel) the load-balance is typically worse.

\section{Recent simulations run with the ART code}

In this section we present some results obtained from a series of recent simulations done with the MPI version of the ART code at different supercomputers. In simulations with $1024^{3}$ particles we identify 1-2 million halos. This is an excellent database for many different kinds of statistics.

Figure 3 shows the evolution of the power spectrum of perturbations in a large simulation of the $\Lambda \mathrm{CDM}$ model: $1 \mathrm{Gpc}$ box with $1024^{3}$ particles. The longest waves in the simulation have small amplitudes and must grow according to the linear theory. This indeed is the case, as seen in the bottom panel. Note, that a small dip $\sim-2 \%$ at $k=0.05-0.07 h^{-1} \mathrm{Mpc}$ is what the quasi-linear theory of perturbations predicts [4]. The plot also shows the main tendency: in the non-linear stage the perturbations at the beginning grow faster than the predictions of the linear theory (we neglect a possible small negative growth extensively discussed in [4]). At later stages the growth slows down, which is seen as bending down of $P(k)$ at high frequencies at $z=0$.

The evolution of the power spectrum at high frequencies (comparable with the Nyquist frequency $k_{\mathrm{Ny}}$ ) is very challenging for N-body codes. Note that the initial power spectrum matches the linear theory nearly perfectly down to $k_{\mathrm{Ny}}$. This is done by perturbing particles from a homogeneous grid. If initial conditions were started with a random distribution, the initial spectrum would have been dominated by the shot noise, whose amplitude would have been $P_{\text {noise }}(k)=1$ : three orders of magnitude higher than the $P(k)$ at $k_{\mathrm{Ny}}$. There is a danger that the high-power fluctuations above $k_{\mathrm{Ny}}$ (discreteness effects) may affect the growth of real low-frequency waves. This does not happen as the comparison of $P(k)$ at $z=65$ and $z=7.7$ shows. At $z=7.7$ the rms density fluctuations are $\delta \rho / \rho \approx 0.4$ and the system is approaching the non-linear stage at high frequencies. Yet, the gradual upturn in the power spectrum seen in the low panel continues all the way to $k \approx 0.8 k_{\mathrm{Ny}}$. This suppression of the discreteness effects is due to a carefully chosen force resolution. Initially we place particles in every other resolution cell. As a result, the code effectively suppreses the discrerteness effects and does not impede the growth of real fluctuations at $k<k_{\mathrm{Ny}}$. At later stages of evolution, when most of small-scale fluctuations have grown and collapsed, we start easing the refinement condition and gradually increase it to normal 2-4 particles per cell. 

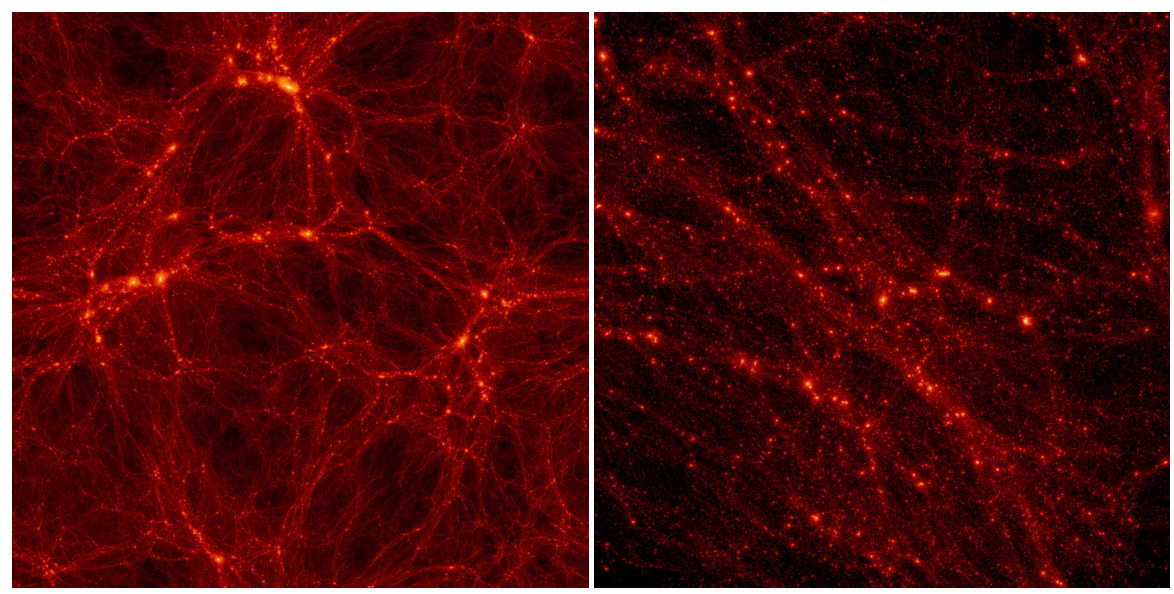

Fig. 4 Left: A slice through a $160 h^{-1} \mathrm{Mpc}$ box with $1024^{3}$ particles. The color codes the dark matter surface density in this $10 \mathrm{Mpc}$ thick slice. Right: Zoom-in to the central $10 h^{-1} \mathrm{Mpc}$ region. The mass of each particle in this re-simulation was 64 times smaller than in the simulation shown in the left panel. A large halo just at the center has a mass $\sim 10^{12} M_{\odot}$. Its environment is similar to the invironment of our galaxy. Note that a large filamentary structure, which goes from the left top corner to the bottom right corner is composed of numerous small filaments. A variety of dark matter haloes is found along and at the intersections of different filaments.

In Fig. 4, left panel, we show an example of the density field in one of the simulations. The density distribution is remarkably complex. There are large quasispherical under-dense regions of different sizes. The dense regions show two types of structures. When the density is very large the structures are nearly spherical (typical axial ratios are 1:1.5 - 1:2). Those are called halos. There are numerous filaments, which have lower density. The filaments contain chains of halos with the largest halos placed at intersections of filaments.

To study in more detail the properties of objects, we also performed high mass resolution simulations of selected regions. The right panel of Fig. 44 shows an example of such a simulation where we selected a sphere of approximately mean density close to the center of the simulation box shown in Fig. 4, left panel. The mass resolution in the low-resolution simulation is $2.6 \times 10^{8} h^{-1} \mathrm{M}_{\odot}$ : the whole $160 h^{-1} \mathrm{Mpc}$ box was simulated with $1024^{3}$ particles. In the re-simulated region of radius $\sim 15 h^{-1} \mathrm{Mpc}$ the mass resolution is $4.0 \times 10^{6} h^{-1} \mathrm{M}_{\odot}$. Therefore, objects similar to the Local Group are resolved with almost 1 million particles. One can see a clear difference between the left and right panels in Fig. 44 in the small region, an environment typical for our Galaxy, there are many tiny filaments pointing in the same direction, which is also the direction of the large-scale velocity field. Hundreds of small halos $\left(10^{8} h^{-1} \mathrm{M}_{\odot}\right.$ to $\left.10^{9} h^{-1} \mathrm{M}_{\odot}\right)$ are strung together along these filaments.

The identification of haloes is always a challenge. We have developed two algorithms: the hierarchical friends-of-friends (HFOF) and the bound density maxima (BDM) algorithms [9]. Both were parallelized using MPI (FOF) or OpenMP (BDM). They are complementary and find essentially the same haloes. Thus, we be- 

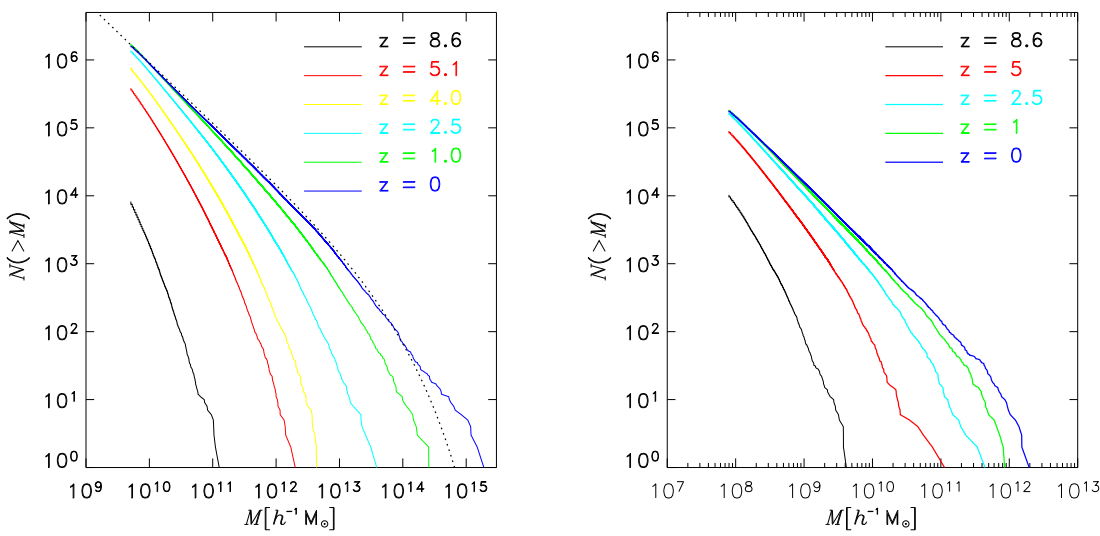

Fig. 5 Left: The mass function of FOF-halos detected at different redshifts in the simulation shown in the left panel of Fig. 4 The dotted curve is analytical approximation [19]. Right: The mass function in the high resolution area shown in the right panel of Fig. 4.

lieve that the algorithms are stable and capable of identifying all dark matter haloes in the simulations. The advantage of the HFOF algorithm is that it can handle haloes of arbitrary shape at arbitrary over-density, not just spherical haloes. The advantage of the BDM algorithm is that it describes the physical properties of the haloes better by identifying and removing unbound particles. This is particularly important for finding sub-halos.

In Fig. 5 we show the mass function of halos detected with the friends-of-friends algorithm. We have identified in the full box already at redshift $z=8.6$ more than 8000 halos and at redshift $z=0$ more than 1.6 million halos (left panel). Due to the better mass resolution in the re-simulation region (a sphere of $15 h^{-1} \mathrm{Mpc}$ at redshift $z=0$ ) we can identify 64 times less massive halos. We found at redshift $z=8.6$ more than 10,000 halos, and at redshift $z=0$ almost 200,000 halos (right panel).

In [17] we used a high resolution re-simulation of a filament with $150,000,000$ particles as well as simulations of a full boxes of 80 and $120 h^{-1} \mathrm{Mpc}$ size with $512^{3}$ particles to study isolated halos. With a mass resolutions of $4.9 \times 10^{6} h^{-1} \mathrm{M}_{\odot}, 3.2 \times$ $10^{8} h^{-1} \mathrm{M}_{\odot}$ or $1.1 \times 10^{9} h^{-1} \mathrm{M}_{\odot}$ density profiles of collapsed galaxy-size dark matter halos with masses $10^{11}-5 \cdot 10^{12} \mathrm{M}_{\odot}$ can be measured very accurately. We found that isolated halos in this mass range extend well beyond the formal virial radius $R_{\mathrm{vir}}$ exhibiting all properties of virialized objects up to $2-3 R_{\mathrm{vir}}$ : relatively smooth density profiles and no systematic infall velocities. Contrary to more massive halos, the dark matter halos in this mass range do not grow through a steady accretion of satellites. For larger radii we combine the statistics of the initial fluctuations with the spherical collapse model to obtain predictions for the mean and most probable density profiles. The model gives excellent results beyond 2-3 formal virial radii.

Based on a simulation of a $150 h^{-1} \mathrm{Mpc}$ box we studied the efficiency of different approaches to interloper treatment in dynamical modeling of galaxy clusters [21]. 
Taking advantage of the full 3D information available from the simulation, we selected samples of interlopers defined with different criteria to assess the efficiency of different interloper removal schemes. We found that the direct methods exclude on average 60-70 percent of unbound particles producing a sample with contamination as low as 2-4 percent. Using indirect approaches, which are applied to the data stacked from many objects, we reproduced the properties of composite clusters and estimated the probability of finding an interloper as a function of distance from the object center. We used mock catalogs extracted from the same simulation to test a new method with which we studied the mass distribution in six nearby $(z<0.06)$ relaxed Abell clusters of galaxies [15]. Based on this cosmological $N$-body simulation we are able to interpret the complex velocity distribution of galaxies in galaxy cluster Abell 1689 [14].

Acknowledgements The computer simulations described here have been performed at LRZ Munich, BSC Barcelona and NAS Ames. We acknowledge support of NSF and NASA grants to NMSU and DAAD support of our collaboration. We thank A. Kravtsov (University of Chicago), G. Yepes (UAM, Madrid), A. Khalatyan (AIP, Potsdam), and Y. Hoffman (HU, Jerusalem) for helpful discussions.

\section{References}

1. Ceverino, D., \& Klypin, A. 2007, ArXiv e-prints, 710, arXiv:0710.1666

2. Colín, P., Klypin, A., Valenzuela, O., \& Gottlöber, S. 2004, ApJ, 612, 50

3. Colín, P., Valenzuela, O., \& Klypin, A. 2006, ApJ, 644, 687

4. Crocce, M., \& Scoccimarro, R. 2008, Phys.Rev.D., 77, 023533

5. Gnedin, N. Y., Kravtsov, A. V., \& Chen, H.-W. 2008, ApJ, 672, 765

6. Gottlöber S., Łokas E. L., Klypin, A., Hoffman, Y., 2003, MNRAS, 344, 715

7. Hockney R. W., Eastwood J. W., 1988, csup.book,

8. Khokhlov, A.M. 1998, J. Comp. Phys. 143, 519

9. Klypin A., Gottlöber S., Kravtsov A. V., Khokhlov A. M.,1999, Astrophys. J. 516, 530

10. Kravtsov, A. V., Klypin, A. A., \& Khokhlov, A. M. 1997, Astrophys. J. Suppl. 111, 73

11. Kravtsov, A. V. 1999, PhD thesis, New Mexico State University

12. Kravtsov, A. V., Klypin, A., \& Hoffman, Y. 2002, ApJ, 571, 563

13. Kravtsov, A. V., Nagai, D., \& Vikhlinin, A. A. 2005, ApJ, 625, 588

14. Łokas E. L., Prada F., Wojtak R., Moles M., Gottlöber S., 2006, MNRAS, 366, L26

15. Łokas E. L., Wojtak R., Gottlöber S., Mamon G. A., Prada F., 2006, MNRAS, 367, 1463

16. Nagai, D., Kravtsov, A. V., \& Vikhlinin, A. 2007, ApJ, 668, 1

17. Prada, F., Klypin, A. A., Simonneau, E., Betancort-Rijo, J., Patiri, S., Gottlöber, S. 2006, Astrophys. J. 645, 1001

18. Rudd, D. H., Zentner, A. R., \& Kravtsov, A. V. 2008, ApJ, 672, 19

19. Tinker, J. L., Kravtsov, A. V, Klypin, A., Abazajian, K., Warren, M. S., Yepes, G., Gottlöber, S., Holz, D. E., 2008, astro-ph:0803.2706

20. Valenzuela, O., \& Klypin, A. 2003, MNRAS, 345, 406

21. Wojtak R., Łokas E. L., Mamon G. A., Gottlöber S., Prada F., Moles M., 2007, A\&A, 466, 437 